\title{
Clinical significance of microRNA-34a in esophageal squamous cell carcinoma
}

\author{
X. Lin, X.Y. Xu, Q.S. Chen and C. Huang \\ Department of Thoracic Surgery, \\ Provincial Clinical College of Fujian Medical University, Fujian Provincial Hospital, \\ Fuzhou, China \\ Corresponding author: $\mathrm{X}$. Lin \\ E-mail: dr_linxing@126.com \\ Genet. Mol. Res. 14 (4): 17684-17691 (2015) \\ Received August 26, 2015 \\ Accepted October 16, 2015 \\ Published December 21, 2015 \\ DOI http://dx.doi.org/10.4238/2015.December.21.41
}

ABSTRACT. MicroRNA-34a (miR-34a) has been found to be downregulated in esophageal squamous cell carcinoma (ESCC) tissues compared with that in normal tissues $(P<0.05)$, and miR-34a overexpression increased apoptosis and decreased clonogenic formation. However, the clinical significance and prognostic value of miR-34a in ESCC has not yet been investigated. In total, 111 patients with ESCC diagnosed and treated at the Department of Thoracic Surgery of Fujian Provincial Hospital between March 2008 and February 2014 were included in this retrospective study. Quantitative real-time PCR was performed to detect expression levels of miR-34a. The associations between miR-34a expression and clinicopathological features were analyzed using $\chi^{2}$ tests. For analysis of survival data, Kaplan-Meier curves were constructed, and the log-rank test was performed. The expression levels of miR-34a in ESCC tissues were significantly decreased $(P<0.01)$, compared with those in adjacent normal esophageal tissues. Low miR-34a expression in ESCC tissues was significantly associated with tumor differentiation $(P=0.013)$, lymph node status $(P=0.038)$, and advanced clinical stage $(P<0.001)$. The KaplanMeier analysis and log-rank test revealed that low miR-34a levels had a 
significant impact on overall survival of patients with ESCC $(P=0.006)$. Multivariate analyses showed that the expression of miR-34a was an independent prognostic factor for ESCC $(P=0.018)$. Our findings indicate that there is reduced expression of miR-34a in human ESCC tissues and suggest a crucial role for miR-34a downregulation in ESCC progression and prognosis.

Key words: MiR-34a; Esophageal squamous cell carcinoma

\section{INTRODUCTION}

Esophageal cancer is the eighth most common cancer and the sixth most common cause of cancer mortality worldwide (Siegel et al., 2015). Esophageal squamous cell carcinoma (ESCC) and adenocarcinoma are the two main forms of esophageal cancer, and both have different etiological and pathological characteristics. To date, ESCC remains the predominant subtype of esophageal cancer. Despite of a variety of treatments, the prognosis of this disease is bleak, with an approximately $10 \% 5$-year survival rate (Song et al., 2014). Therefore, it is important to identify biological markers of ESCC to improve early diagnosis and develop new treatment strategies to combat the disease.

MicroRNAs (miRNAs) are a class of small non-coding RNAs of approximately 2022 nucleotides that play key roles in development and in various biological processes such as cell differentiation, proliferation, oncogenesis, angiogenesis, and cell invasion through posttranscriptional regulation of gene expression (Ambros, 2004; Zhang and Su, 2009). Moreover, there is growing evidence that the expression of miRNAs plays an important role in the progress of esophageal cancer (Chu et al., 2013; Hong et al., 2014; Huang et al., 2014).

The microRNA-34 (miR-34) family consists of miR-34a, miR-34b, and miR-34c, with miR34 a belonging to the p53 tumor suppressor network (Cha et al., 2012; Wang et al., 2014). It has been demonstrated that the miR-34 family directly links tumor suppressor functions and oncogenic pathways in some cancers (Cha et al., 2012; Wang et al., 2014). miR-34a is a direct proapoptotic transcriptional target of p53 that can mediate some of its biological effects (Raver-Shapira et al., 2007). miR-34a is commonly deleted in various types of cancers including breast, lung, pancreatic, and bladder cancers (Bader, 2012). The downregulated expression of miR-34a-controlled genes may occur due to mutations that inactivate p53 in tumor cells (Balca-Silva et al., 2012). Recently, Nie et al. (2015) found that miR-34a was downregulated in ESCC tissues compared with that in normal tissues $(P<0.05)$, and that miR-34a overexpression increased apoptosis and decreased clonogenic formation, whereas it inhibited invasion and migration of ESCC cells by suppressing MMP-2 and -9 expression. However, the clinical significance and prognostic value of miR-34a in ESCC had not yet been investigated.

\section{MATERIAL AND METHODS}

\section{Patients and tissue samples}

The Ethics Committee of Provincial Clinical College of Fujian Medical University, Fujian Provincial Hospital, permitted the use of tissue sections for research purposes in the current study. Moreover, signed informed consent from all the patients was obtained. In total, 111 
patients with ESCC diagnosed and treated at the Department of Thoracic Surgery of Fujian Provincial Hospital between March 2008 and February 2014 were included in the retrospective database for analysis herein. All patients' clinicopathological information had been previously recorded and the specimens had been collected. Additionally, all patients had not received any radiotherapy or chemotherapy prior to the surgical procedure performed. TNM classifications after surgery were made according to the International Union Against Cancer (UICC) staging criteria for esophageal cancer. Table 1 summarizes the clinicopathological characteristics of all 111 ESCC patients enrolled in this study.

\section{RNA extraction and quantitative real-time PCR}

Total cellular RNA was extracted using the TRIzol Reagent (Invitrogen, Carlsbad, CA, USA) according to manufacturer instructions. Quantitative real-time PCR (qRT-PCR) was performed to detect expression levels of miR-34a in 111 self-paired specimens of ESCC and adjacent normal esophageal tissues. Then, $1 \mu \mathrm{g}$ total RNA was reverse transcribed into complementary DNA (cDNA) by using the miScript Reverse Transcription Kit (QIAGEN, Valencia, CA, USA). The 20- $\mu$ L PCR system consisted of $10 \mu \mathrm{L} 2 \mathrm{X}$ QuantiTect SYBR Green PCR Master Mix, $2 \mu \mathrm{L}$ 10X miScript Universal Primer, $300 \mathrm{nM}$ forward primer, $1 \mu \mathrm{L}$ cDNA, and RNase-free water (QIAGEN). The reactions were incubated on 96 -well optical plates at $95^{\circ} \mathrm{C}$ for $6 \mathrm{~min}$, followed by 30 cycles of $95^{\circ} \mathrm{C}$ for $20 \mathrm{~s}$ and $56^{\circ} \mathrm{C}$ for $10 \mathrm{~s}$. The expression of the miRNA was defined based on the threshold cycle

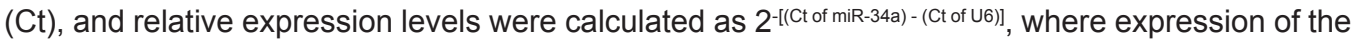
U6 small nuclear RNA was used as the internal control.

\section{Statistical analysis}

Statistical analysis was performed with the SPSS software (SPSS Standard version 19.0, SPSS, Chicago, IL, USA). The associations between miR-34a expression and clinicopathological features were analyzed using $\chi^{2}$ tests. For analysis of survival data, Kaplan-Meier curves were constructed, and the log-rank test was performed. Overall survival (OS) was calculated as the time from the date of surgery to the date of death or final clinical follow-up. Patients who were lost to follow-up were considered to be censored. Univariate and multivariate analyses were performed using the Cox proportional hazard model to evaluate the correlation between miR-34a expression and patient prognosis. A $P$ value $<0.05$ was considered to be statistically significant.

\section{RESULTS}

\section{Decreased expression of miR-34a in ESCC tissues}

The expression levels of miR-34a in ESCC tissues were significantly decreased compared to those in adjacent normal esophageal tissues $(P<0.01$; Figure 1). The median value of miR-34a expression in ESCC tissues detected via qRT-PCR was used as a cutoff point to classify all 111 ESCC patients into low and high miR-34a expression groups. ESCC patients expressing miR-34a at levels less than the median expression level were assigned to the low expression group $(\mathrm{N}=$ 53), and those expressing miR-34a at levels equal to or above the median value were assigned to the high expression group $(\mathrm{N}=58)$. 


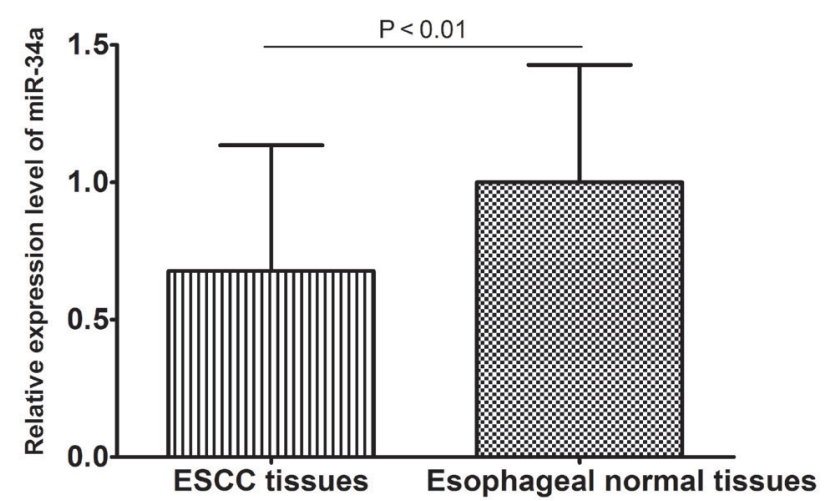

Figure 1. Decreased expression of miR-34a in esophageal squamous cell carcinoma tissues compared with that in normal esophageal tissues.

\section{Relationships between clinicopathological features and miR-34a expression}

Table 1 summarizes the associations between miR-34a expression and clinicopathological features of ESCC patients. Low miR-34a expression in ESCC tissues was significantly associated with tumor differentiation $(P=0.013)$, lymph node status $(P=0.038)$, and advanced clinical stage $(P<0.001)$. However, there were no significant associations between miR-34a expression and patient age, gender, smoking status, tumor location, or T stage $(P>0.05)$.

\begin{tabular}{|c|c|c|c|c|}
\hline \multirow[t]{2}{*}{ Characteristic } & \multirow[t]{2}{*}{ Case number } & \multicolumn{2}{|c|}{ miR-34a expression } & \multirow[t]{2}{*}{$P$ value } \\
\hline & & $\operatorname{Low}(\mathrm{N}=53)$ & High $(N=58)$ & \\
\hline \multicolumn{5}{|l|}{ Age (years) } \\
\hline$<55$ & 43 & 17 & 26 & \multirow[t]{2}{*}{0.179} \\
\hline$\geq 55$ & 68 & 36 & 32 & \\
\hline \multicolumn{5}{|l|}{ Gender } \\
\hline Male & 61 & 28 & 33 & \multirow[t]{2}{*}{0.706} \\
\hline Female & 50 & 25 & 25 & \\
\hline \multicolumn{5}{|l|}{ Smoking history } \\
\hline Yes & 66 & 36 & 30 & \multirow[t]{2}{*}{0.121} \\
\hline No & 45 & 17 & 28 & \\
\hline \multicolumn{5}{|l|}{ Site of tumor } \\
\hline Upper thoracic & 35 & 15 & 20 & \multirow[t]{3}{*}{0.598} \\
\hline Middle thoracic & 51 & 27 & 24 & \\
\hline Lower thoracic & 25 & 11 & 14 & \\
\hline \multicolumn{5}{|l|}{ Differentiation } \\
\hline Good & 43 & 19 & 24 & \multirow[t]{3}{*}{0.013} \\
\hline Moderate & 47 & 18 & 29 & \\
\hline Poor & 21 & 16 & 5 & \\
\hline \multicolumn{5}{|l|}{ T stage } \\
\hline $\mathrm{T} 1-2$ & 72 & 31 & 41 & \multirow[t]{2}{*}{0.233} \\
\hline T3-4a & 39 & 22 & 17 & \\
\hline \multicolumn{5}{|l|}{ Lymph node status } \\
\hline Yes & 33 & 21 & 12 & \multirow[t]{2}{*}{0.038} \\
\hline No & 78 & 32 & 46 & \\
\hline \multicolumn{5}{|l|}{ TNM stage } \\
\hline $1+\mid 1$ & 69 & 22 & 47 & \multirow[t]{2}{*}{$<0.001$} \\
\hline III & 42 & 31 & 11 & \\
\hline
\end{tabular}




\section{miR-34a expression in primary tumors predicts ESCC patient outcome}

To investigate whether miR-34a could serve as a prognostic marker for ESCC, KaplanMeier analysis and log-rank test were performed to assess the association between miR34a expression and 5-year overall survival. The results showed that low miR-34a levels had a significant impact on OS of patients with ESCC ( $P=0.006$; Figure 2). Multivariate analyses were performed with the Cox proportional hazard model. The expression of miR-34a $(P=0.018)$, tumor differentiation $(P=0.021)$, and TNM stage $(P=0.003)$ were independent prognostic factors for patients with ESCC (Table 2).

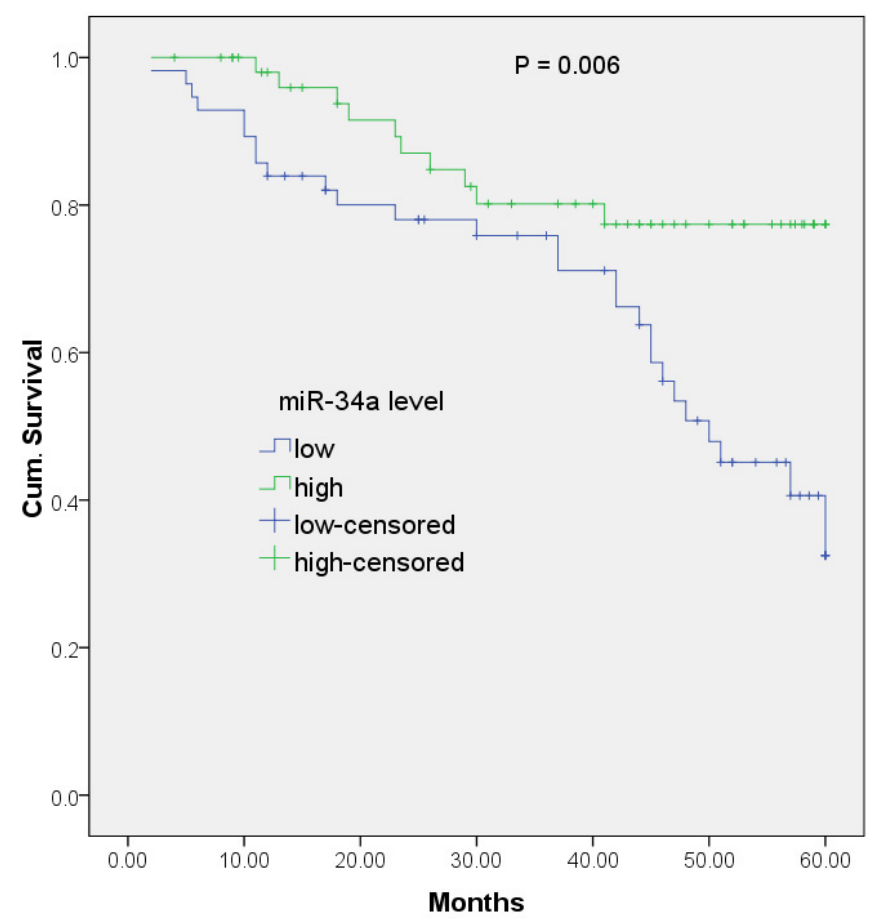

Figure 2. Kaplan-Meier analysis and log-rank test showed that low miR-34a level had a significant impact on overall survival of patients with esophageal squamous cell carcinoma $(P=0.006)$.

Table 2. Multivariate Cox hazard model analysis for prognostic factors.

\begin{tabular}{lccr}
\hline Variable & & Multivariate analysis \\
\cline { 2 - 4 } & Hazard ratio & $95 \% \mathrm{Cl}$ & $\mathrm{P}$ value \\
\hline Age & 1.028 & $0.273-2.118$ & 0.271 \\
Gender & 0.774 & $0.363-2.293$ & 0.725 \\
Smoking history & 2.192 & $0.682-4.182$ & 0.126 \\
Site of tumor & 0.782 & $0.227-2.291$ & 0.362 \\
Differentiation & 2.195 & $1.882-9.273$ & 0.021 \\
T stage & 1.341 & $0.782-3.914$ & 0.089 \\
Lymph node status & 3.192 & $0.923-5.182$ & 0.051 \\
TNM stage & 4.105 & $3.021-12.991$ & 0.003 \\
miR-34a level & 3.182 & $1.273-10.184$ & 0.018 \\
\hline
\end{tabular}




\section{DISCUSSION}

Although ESCC is relatively uncommon among cancers, its incidence has been reportedly increasing in recent years and varies between developed and developing counties. China has the highest incidence of ESCC, which is the fourth highest cause of cancer mortality (Lin et al., 2015). Despite advances in diagnosis and multimodal therapies, the prognosis for patients with ESCC remains poor, with a 5 -year survival rate of less than $15 \%$ for advanced staged cancers (Thallinger et al., 2011). Therefore, innovative strategies derived from a better understanding of ESCC biology are needed to further improve the outcome of patients with this disease.

miRNAs can act as either oncogenes or tumor suppressor genes and play vital roles in cancer development and cellular processes including proliferation, apoptosis, and migration. Furthermore, it has been demonstrated that the abnormal expression of miRNAs is associated with the development and progression of cancer, and has prognostic significance for ESCC. Therefore, miRNAs may serve as new biomarkers for ESCC (Ratner et al., 2010; Zhao et al., 2013).

The miR-34 family is comprised of three members (miR-34a, miR-34b, and miR-34c) that are part of the p53 network, and whose expressions are directly induced by p53 in response to DNA damage or oncogenic stress. miR-34a is located on chromosome 1 p36 and acts as a potent tumor suppressor gene in a p53-dependent or -independent manner (Hermeking, 2010). Previous studies have reported that several key molecules were identified as targets of miR-34a, including Bcl-2, transcriptional repressor protein YY1, Notch1, and Notch2 (He et al., 2007; Li et al., 2009; Chen et al., 2011). The prognostic value of miR-34a has been investigated in several cancers. Gallardo et al. (2009) identified miR-34a as a novel prognostic marker in non-small-cell lung cancer (NSCLC) patients, providing a potential method for estimating a patient's risk of disease recurrence and a useful tool to help guide treatment decisions. Gao et al. (2013) found that miR-34a expression levels positively correlated with tumor WHO grades of glioma. Additionally, in patients with grade III and IV gliomas, lower miR-34a expression correlated with worse progression-free survival and overall survival. Univariate and multivariate analyses further revealed that miR-34a was an independent prognostic indicator for glioma. Therefore, miR-34a expression was a novel and potentially useful signature for predicting glioma prognosis (Gao et al., 2013). In a study by Jin et al. (2014), significantly lower miR-34a expression was observed in gallbladder adenocarcinoma tissues compared to that of healthy gallbladders, which correlated with poor prognosis of patients with gallbladder adenocarcinoma. Zhang et al. (2015) found that levels of miR-34a expression were significantly decreased in gastric cancer tissues compared with adjacent normal tissues $(P<0.001)$. Additionally, low miR-34a expression was associated with lymph node involvement $(P=0.004)$, advanced TNM stage $(P=0.006)$, poor tumor differentiation $(P=0.024)$, high tumor recurrence rate $(P=0.008)$, and poor 5-year survival $(P<0.001)$. Furthermore, when analyzed with a multivariate Cox regression model, low miR-34a levels were significantly correlated with high recurrence rates and poor overall survival. Taken together, their results suggested that downregulation of miR-34a in gastric cancer was associated with high recurrence and poor overall survival, and that miR-34a may serve as a prognostic marker for gastric cancer (Zhang et al., 2015). However, the clinical significance and prognostic value of miR-34a had not been investigated in ESCC.

In the present study, we found that the expression levels of miR-34a in ESCC tissues were significantly decreased compared with those in adjacent normal esophageal tissues. Low miR-34a expression in ESCC tissues was significantly associated with tumor differentiation, lymph node status, and advanced clinical stage. To investigate whether miR-34a could serve as a prognostic maker for ESCC, Kaplan-Meier analysis and log-rank test were performed to assess the association 
between miR-34a expression and the 5-year overall survival. The results showed that low miR-34a levels had a significant negative impact on OS in patients with ESCC. Multivariate analyses were performed with the Cox proportional hazard model, which revealed that the expression of miR-34a, tumor differentiation, and TNM stage were independent prognostic factors for patients with ESCC.

In conclusion, our findings show that there is reduced expression of miR-34a in human ESCC tissues, which suggests a crucial role for miR-34a downregulation in ESCC progression and prognosis.

\section{Conflicts of interest}

The authors declare no conflict of interest.

\section{REFERENCES}

Ambros V (2004). The functions of animal microRNAs. Nature 431: 350-355.

Bader AG (2012). miR-34 - a microRNA replacement therapy is headed to the clinic. Front. Genet. 3: 120.

Balca-Silva J, Sousa Neves S, Goncalves AC, Abrantes AM, et al. (2012). Effect of miR-34b overexpression on the radiosensitivity of non-small cell lung cancer cell lines. Anticancer Res. 32: 1603-1609.

Cha YH, Kim NH, Park C, Lee I, et al. (2012). miRNA-34 intrinsically links p53 tumor suppressor and Wnt signaling. Cell Cycle 11: 1273-1281.

Chen QR, Yu LR, Tsang P, Wei JS, et al. (2011). Systematic proteome analysis identifies transcription factor YY1 as a direct target of miR-34a. J. Proteome Res. 10: 479-487.

Chu Y, Zhu H, Lv L, Zhou Y, et al. (2013). miRNA s in oesophageal squamous cancer. Neth. J. Med 71: 69-75.

Gallardo E, Navarro A, Vinolas N, Marrades RM, et al. (2009). miR-34a as a prognostic marker of relapse in surgically resected non-small-cell lung cancer. Carcinogenesis 30: 1903-1909.

Gao H, Zhao H and Xiang W (2013). Expression level of human miR-34a correlates with glioma grade and prognosis. J. Neurooncol. 113: 221-228.

He L, He X, Lim LP, de Stanchina E, et al. (2007). A microRNA component of the p53 tumour suppressor network. Nature 447: 1130-1134.

Hermeking $\mathrm{H}$ (2010). The miR-34 family in cancer and apoptosis. Cell Death Differ. 17: 193-199.

Hong L, Han Y, Zhang H, Zhao Q, et al. (2014). Prognosis-related microRNAs in esophageal cancer. Expert Opin. Biol. Ther. 14: 483-489.

Huang J, Zhang SY, Gao YM, Liu YF, et al. (2014). MicroRNAs as oncogenes or tumour suppressors in oesophageal cancer: potential biomarkers and therapeutic targets. Cell Proliferat. 47: 277-286.

Jin K, Xiang Y, Tang J, Wu G, et al. (2014). miR-34 is associated with poor prognosis of patients with gallbladder cancer through regulating telomere length in tumor stem cells. Tumour Biol. 35: 1503-1510.

Li Y, Guessous F, Zhang Y, Dipierro C, et al. (2009). MicroRNA-34a inhibits glioblastoma growth by targeting multiple oncogenes. Cancer Res. 69: 7569-7576.

Lin L, Wang J, Jiang Y, Meng N, et al. (2015). Interstitial 125I seed implantation for cervical lymph node recurrence after multimodal treatment of thoracic esophageal squamous cell carcinoma. Technol. Cancer Res. Treat. 14: 201-207.

Nie J, Ge X, Geng Y, Cao H, et al. (2015). miR-34a inhibits the migration and invasion of esophageal squamous cell carcinoma by targeting Yin Yang-1. Oncol. Rep. 34: 311-317.

Ratner ES, Tuck D, Richter C, Nallur S, et al. (2010). MicroRNA signatures differentiate uterine cancer tumor subtypes. Gynecol. Oncol. 118: 251-257.

Raver-Shapira N, Marciano E, Meiri E, Spector Y, et al. (2007). Transcriptional activation of miR-34a contributes to p53mediated apoptosis. Mol. Cell 26: 731-743.

Siegel RL, Miller KD and Jemal A (2015). Cancer statistics, 2015. CA: Cancer J. Clin. 65: 5-29.

Song Y, Li L, Ou Y, Gao Z, et al. (2014). Identification of genomic alterations in oesophageal squamous cell cancer. Nature 509: 91-95.

Thallinger CM, Raderer M and Hejna M (2011). Esophageal cancer: a critical evaluation of systemic second-line therapy. J. Clin. Oncol. 29: 4709-4714.

Wang AM, Huang TT, Hsu KW, Huang KH, et al. (2014). Yin Yang 1 is a target of microRNA-34 family and contributes to gastric carcinogenesis. Oncotarget 5: 5002-5016.

Zhang H, Li S, Yang J, Liu S, et al. (2015). The prognostic value of miR-34a expression in completely resected gastric cancer: 
tumor recurrence and overall survival. Int. J. Clin. Exp. Med. 8: 2635-2641.

Zhang R and Su B (2009). Small but influential: the role of microRNAs on gene regulatory network and 3'UTR evolution. J. Genet. Genomics = Yi chuan xue bao 36: 1-6.

Zhao BS, Liu SG, Wang TY, Ji YH, et al. (2013). Screening of microRNA in patients with esophageal cancer at same tumor node metastasis stage with different prognoses. Asian Pac. J. Cancer Prev. 14: 139-143. 